\title{
A Reliable Framework for Communication in Internet of Smart Devices using IEEE 802.15.4
}

\author{
Tanweer Alam ${ }^{1}$ \\ ${ }^{1}$ Islamic University of al-Madinah al-Munawarah
}

June 8, 2020

How to cite this article?

Tanweer Alam. " A Reliable Framework for Communication in Internet of Smart Devices using IEEE 802.15.4.", ARPN Journal of Engineering and Applied Sciences. Vol 13(10). 2018.

\begin{abstract}
Peoples are naturally communicators but devices are not. In the Internet of Things (IoT) architecture, the smart devices (SDs), sensors, programs and association of smart objects are connected together to transfer information among them. The SD is designed as physical device linked with computing resources that are capable to connect and communicate with another SD through any medium and protocol. The communication among intelligent physical things is a challenging task to exchange information that guaranteed to reach to the destination completely in a real time with the same order as sending without corruption. The reliable communication between physical things can be built in the transmission control protocol (TCP) layers. In TCP layer, the reliable communication is required the error detection, correction and confirmation to exchange information among smart devices. In this paper, the author represents a framework to deal with reliability issues to enable the adoption of IoT devices. The results found the improvement in reliability.
\end{abstract}

Keywords: Reliability, Wireless Communication, Internet of Things, Transmission control protocol, Sensor Networks.

\section{Introduction}

Now-a-days, in whole world the smart devices are increasing exponentially. They provide much more facility to the end users and also attach with their daily life. Currently mobiles are using the centralized cell phone network for personal communication within the last decade. Smart phone is designed technically to make phone very useful for the end users. Now we can send text, pictures, voice and video to each other using strong cellular networks. Smart phone also can connect to the internet easily for sending and receiving data within the cellular network. The Internet of Things describes a network of smart objects where they can communicate and share the information among each other using Internet. The smart objects are intelligent devices with inbuilt software, sensor and programs. Every smart object has a unique identification in network by their internal programs. Figure 1 shows that the internet of smart devices network is the combination of smart device applications and layered framework included IEEE 802.15.4. 


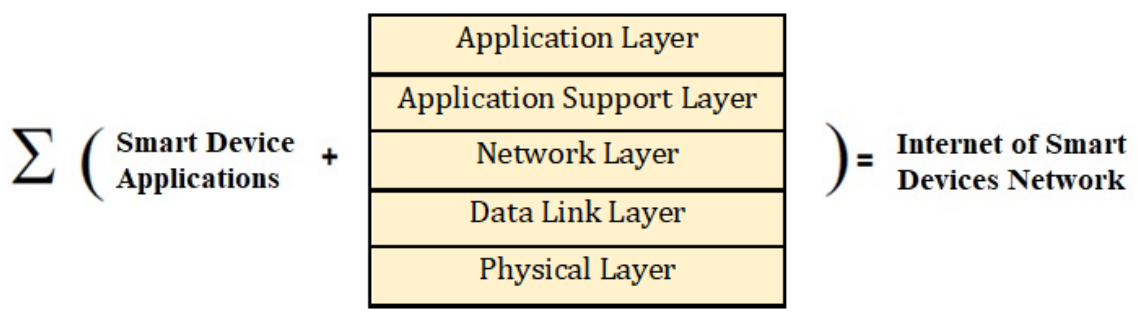

Fig.1: Internet of smart device network

The reliability is the main issue in interconnected environments where many sensors, actuators, controllers and intelligent objects etc. are connected. The smart devices are not just smart phones, it may be smart refrigerator, Smart home automation entry point, smart air conditioners, Smart hubs, Smart thermostat, Color changing smart LEDs, Smart Watches and smart Tablets etc. in internet of things framework they are connected to each other through internet. The proposed research plan builds research on extending reliability for communication in smart devices using IEEE802.15.4. The transfer data from one configuration to another using wireless networks starts from 1973 in the form of packets radio network. They were able to communicate with another same configuration devices. Recent work is continuing on a project called the Serval Project. It provides networks facility to android devices for communication in infrastructure less network. Whereas our research is concerned about reliable communication in internet of smart devices. The main contribution of this research is the creation of the communication framework and provide reliable communication using IEEE802.15.4 among the internet of smart devices. The previous studies have been focused on the creation and optimization the framework for communication, but such research doesn't perform the full framework for reliable communication among internet of smart devices. Figure 2 represents the IoT node with reliability feature. 


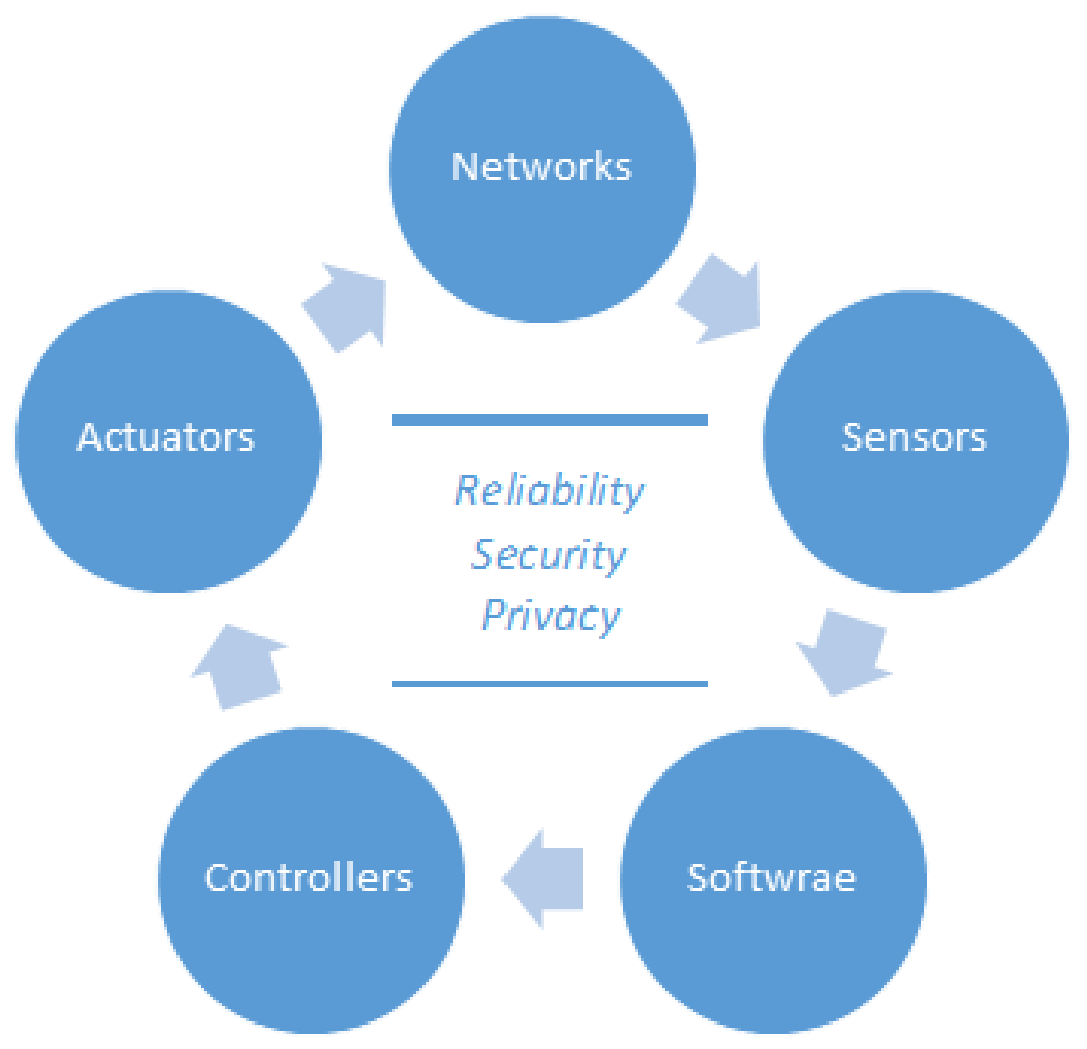

Fig. 2: IoT node with reliability

The proposed research is a step forward in wireless networking and IoT where we propose new reliability framework based on IEEE802.15.4 for communicating in internet of smart devices. The Wireless communication is the key of Internet of things. In the 1980s, with the evolving of the internet, the foundation of an emerging grid computation was established. The foundation involved various principals which employ the internet in a way in which users are provisioned as resource nodes. A grid coordinates these resources nodes and dispenses takes to them thus the entire computation is viewed as a cumulative fashion. The principles paved the way of a novel computing paradigm which eventually carved today's distribution concepts. In the 1990s, the concept of virtualization was driven to the application tier. It followed by employing virtualized private network connections which share the same physical channel. The proposed research work in this project is an enhancement and implementation of Markov-chain and MAC level framework for reliable communication based on IEEE802.15.4 in the framework of internet of things. The research outcome is to establish a new framework for reliable communication in internet of smart devices. The proposed research uses the correct and efficient simulation of a desired steady, and can be implemented in a framework of Internet of Things. In the future, researchers can enhance this research and implement in the internet of everything framework, haptic communication network framework or in the framework of tactile internet. The problem is to establish a new framework for reliable communication among internet of smart devices. The questions are "How is it possible to create a new framework for reliable communication", "can we implement Markov chain model in this research", "How to integrate Markov-chain to MAC level framework". The mostly used wireless sensor network communication protocol for low power consumption is IEEE802.15.4 because this is developed specially for low power, low cost wireless communication and it uses MAC-level. So, it is best technology for reliable communication among internet of smart devices. The IEEE802.15.4 standard offers very low rate and velocity in the communication among internet of smart devices. It focuses on low cost connection with nearby smart devices using infrastructure less network. 
The objective of this research is to create the new model for reliable communication among internet of smart devices. This research is based on IEEE802.15.4 standard with Markov chain and MAC layer. The framework can provide quality of services (QoS) through reducing the traffic rate vacillation also the variety of the quantity of smart devices. In this research we consider idle state in order to makes our examination more efficient, at that point the general execution regarding the overall performance of the framework is evaluated. Based on evaluation the reliability will examine. The evaluation will depend on two points the delay and reliability in internet of smart devices. The reliable framework is essential to reach the QoS level where the evaluation is based on delay and reliability parameters. Device to device slowness refers to the duration when a packet is generated to when a packet service is accomplished, including the time when in the queue as well as transmission time. Here I investigate the time duration of IEEE802.15.4 standard consist of MAC-parameters as well as impact likelihood using the Probability Density Function of transmission time, we designed an optimum network meeting our QoS requirements. Future work includes investigating the performance of proposed framework. The following are the key points- 1 . The research is primarily focused on reliability. It enables smart devices to communicate with another device among internet of smart devices reliably. 2. The proposed framework for reliable communication will access across the internet of smart devices. 3. The results of proposed research will have compared with previous study in the same area.

\section{Research Methodology}

The main contribution of this research is designing a reliable framework for IEEE 802.15.4 standard. The proposed framework is specifically appropriate for applications in which data is periodically transmitted in internet of smart devices environment. In these applications, on one hand, packets are being produced based on a certain periodic time pattern. On the other hand, service time is always a random variable with general distribution. Therefore, service time might temporarily exceed the period time which, as an inevitable consequence some packets might encounter a busy channel and be dropped. We solve this problem by proposing our MAC-level queue. We demonstrate that proposed MAC-level queue, not only increases the throughput, but also the direct connection between the generation (sensors) and communication packet systems are eliminated which make the system far more stable. Moreover, in order to enhance the proposed model, we have employed retransmission scheme, variable packet length, and saturated traffic condition. The solution of this research is summarized as follows. The implementation of reliable framework for communication among internet of smart devices in $5 \mathrm{G}$ will be programmed to execute on to the internet of things. The Smart device must have in built IEEE802.15.4 standard. The idea will implement into threelayer model that layers are DLL, Routing Protocol and Application Layer. DLL is used to create connection between smart devices. Routing protocol handles routing. Application layer is used to handle the activation of proposed framework to communicate in internet of smart devices. The proposed study supports the wireless networking in DLL to establish a framework among internet of smart devices. We prefer to choose Android operating system based smart devices, the reason behind this approach is that android operating system is an open source that is freely available on internet.

\section{Reliability framework in Internet of smart devices}

The reliability in internet of smart devices is refer as the probability that the information is delivered from one smart device to another device successfully. The communication system may be designed like the network flow diagram with group of vertices and edges. The reliability of the devices and links in the network can be obtained using the following equation.

$\mathrm{R}(\mathrm{N})=\{\mathrm{D}, \mathrm{L}\}$ where $\mathrm{D}=\left\{\mathrm{D}_{\mathrm{i}}(\mathrm{N})\right\}$ and $\mathrm{L}=\left\{\mathrm{L}_{\mathrm{i}}(\mathrm{N})\right\} \ldots \ldots(1)$

$\mathrm{R}$ is represented the reliability in communication, $\mathrm{D}$ represents the devices in the network, $\mathrm{L}$ represents the communication link among devices and $\mathrm{N}$ represents the network.

The study to calculate the reliability of the communication among smart devices is to study the delay of delivering the information with the links. The communication links between two devices may be more than one. Suppose Di and Dj are two devices that are connected each other with the links $\mathrm{L}_{1}, \mathrm{~L}_{2}, \ldots . \mathrm{L}_{\mathrm{i}}, \ldots$. $\mathrm{L}_{\mathrm{k}}$. The figure 3 represents the communication link among the smart devices. 


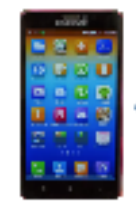

$\mathbf{D}_{\mathrm{i}}$

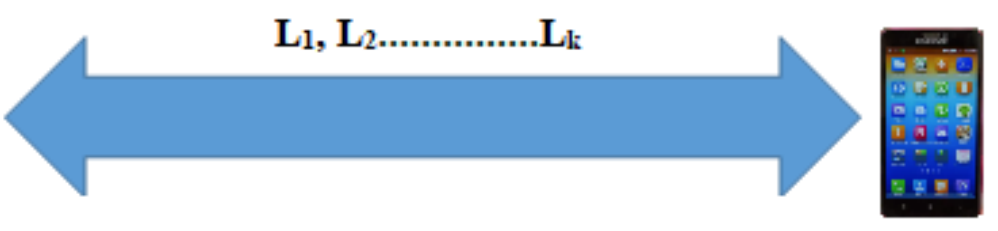

$\mathbf{D}_{\mathrm{j}}$

Fig. 3: Links among smart devices

$$
L_{\mathrm{ij}}(N)=\sum_{k=1}^{n} P_{k} L_{\mathrm{ijk}}(N)
$$

Using equation (2) we can calculate the delay in links. Suppose we have two links $\mathrm{L}_{1}$ and $\mathrm{L}_{2}$ between smart devices. Probability for $\mathrm{L}_{1}$ is $\mathrm{P}_{1}$ and $\mathrm{L}_{2}$ is $\mathrm{P}_{2}$. Consider $\mathrm{P}_{1}=0.5$ and $\mathrm{P}_{2}=0.5$.

The distributed function for the delay can be obtained from the following equation.

$$
\mathrm{L}_{i j}(\mathrm{~N})=\frac{1}{2} \cdot \frac{1}{\mathrm{~N}+\lambda_{i j 1}}+\frac{1}{2} \cdot \frac{1}{\mathrm{~N}+\lambda_{i j 2}} .
$$

\section{..$(3)$}

The reliability of successfully transmitted probability can be obtained from the following equation.

$\mathrm{P}(\mathrm{L})=\mathrm{P}\left\{\mathrm{L}_{\mathrm{i}}\right\}=\sum_{i \in R(N)} \mathrm{Pi}$

Or we can say-

$\mathrm{P}_{\mathrm{k}}=\max \left\{\mathrm{P}_{\mathrm{i}}\right\}$. qq

Or

$\mathrm{P}_{\mathrm{k}}=\min \left\{\frac{1}{P i}\right\}$

$\sum P($ Transmit $)=\sum P($ Successful $)+\sum P($ Unsuccessful $)$.

Figure 4 is representing the reliable connection between two smart devices. R1, R2 and R3 devices are the reliable devices and others are normal devices.

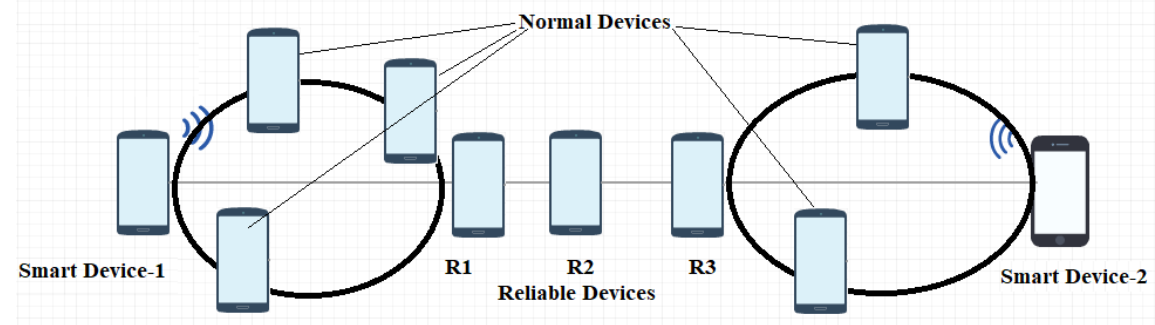

Fig. 4: Reliable connection between two smart devices

The communication among smart devices will be successful all the time if the transmission among smart devices doesn't have any collision or error. According to the article [1], the transmission probability is calculated in the following equation. 
$\frac{d}{\mathrm{dt}} \chi=\delta \sum_{j} \mu_{t}(j)\left[1-\frac{\mu_{t}(0)}{\sum_{j} \mu_{t}(j)}\right]=\delta \sum_{j>0} \mu_{t}(j), t \geq 0$

Where? is the Markovian process at time t. $\chi=\frac{\chi}{n}$.

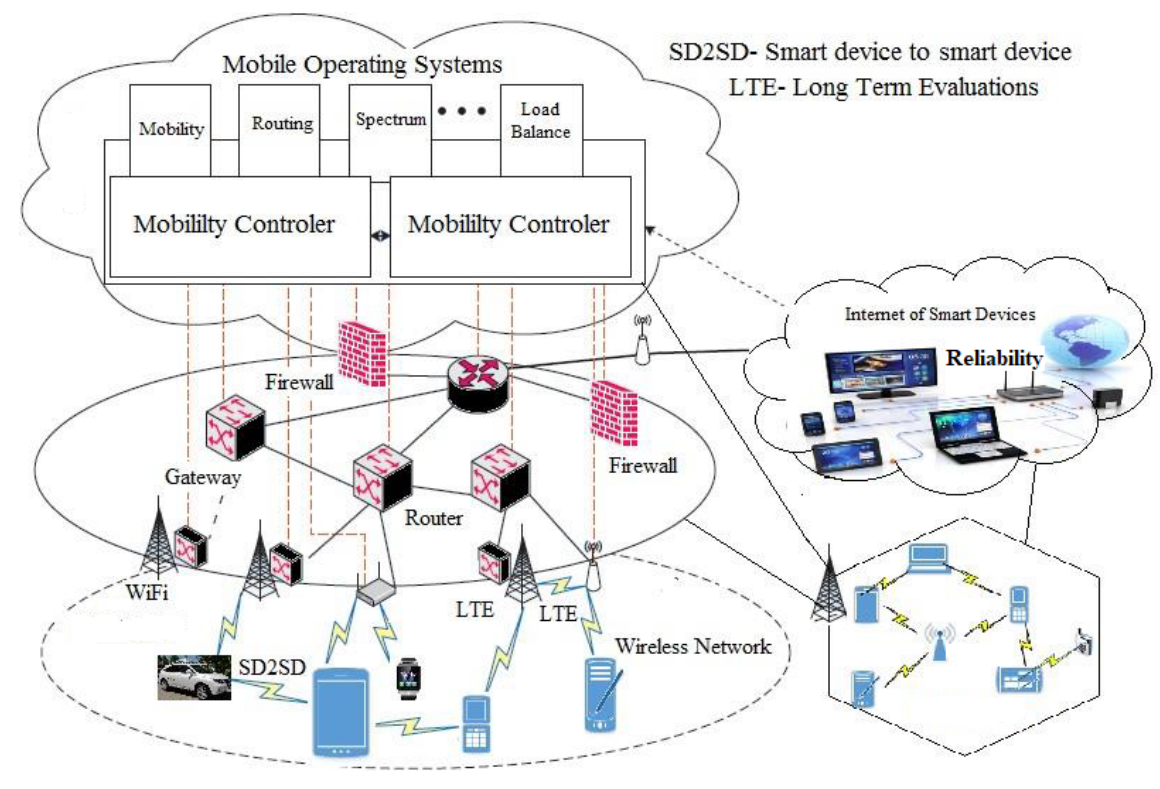

Fig. 5: Reliability in internet of smart devices

The information can transmit from one device to another using information delivers in between. The tempting node of a Markovian network deliver the information among internet of smart devices. It has probability 0. The tempting Markovian network has at least one tempting node. Suppose P1 is the probability of successfully transmission information among internet of smart devices. So, 1-P1 will be the probability of not transmitted packets of information. The idle state probabilities can be obtained by the following equations.

The following algorithm will compute the reliability of communication among Internet of smart devices.

Input: Number of smart devices, transmission function value $\left(\epsilon_{\kappa}\right)$.

Output: Reliability

Initialization: Counter $=0, \mathrm{R}=0$.

Step 1: Find all probabilities of smart devices in each direction.

Step 2: Find entropy per symbol.

Step 3: Find Transmission in bits/sec.

Step 4: Find Reliability

Step 5: Counter $=$ Counter +1 ;

if counter i Number of devices go to step 2 otherwise stop.

The complexity of the algorithm is $O\left(n^{2}\right)$.

The Proposed framework had been implemented using three mobile applications. These mobile applications are verified on three Samsung devices. One of them is supported by $4 \mathrm{G}$ network and another two are supported by $3 \mathrm{G}$ networks. 
The transmission $\left(t_{s}\right)$ of information $\left(I_{k}\right)$ among the number of smart devices $\left(S_{n}\right)$ can be estimated during the time interval $\left[t_{i}, t_{i-1}\right]$. The smart devices can move within the range and access the service using the multidimensional function $\left(\epsilon^{\kappa}\right)$.

$\epsilon \mathcal{K}=C^{S n \times t k} \times I k$

where $\mathrm{k}=0,1,2,3 \ldots \ldots[?](+v e)$.

If smart devices have moved outside the range then $\mathrm{k}$ will be a negative value. Here we consider that the transformation of information happens simultaneously. We know that the probability is proportional to the one divide by information.

$$
k \propto \frac{1}{I_{k}}
$$

P $\ldots \ldots \ldots(10)$

The probability density function for transmission is calculated mathematically as follows.

$\mathrm{P}_{k}\left(S_{n} \mid \epsilon_{\kappa}, I_{\kappa}, \tau_{\kappa}\right)=1-\int_{-\infty}^{\infty} M_{Q}\left[\left(\sqrt{\left(\frac{2 \gamma^{2}}{1-\gamma^{2}} * \frac{S_{n}}{t_{n}}\right)}\right),\left(\sqrt{\left.\left.\left(\frac{S_{n}}{1-\gamma^{2}} * 2 \gamma\right)\right)\right]}\right.\right.$.

where $\gamma[?] 1$

Now we have divided the probability density function of all the connections using the entropy per symbol of all connected devices in 3-dimensional directions.

$P_{x_{k}} * P_{y_{k}} * P_{z_{k}} * \frac{1}{P_{x_{k}}} \cdot \frac{1}{P_{y_{k}}} \cdot \frac{1}{P_{z_{k}}} \ldots \ldots \ldots$

$H_{\alpha, \delta, \epsilon}=\sum_{x=0}^{\infty} \sum_{y=0}^{\infty} \sum_{z=0}^{\infty} \alpha \beta^{-1} \varepsilon_{2}\left(\frac{\delta}{\alpha}, S_{k}, \rho\right) \ldots \ldots \ldots$

Here $\varepsilon_{2}\left(\frac{\delta}{\alpha}, S_{k}, \rho\right)$ is the Chi-Square distribution method that is used here for convergence. Now we will calculate all the probabilities, entropies in each direction and finally, we draw the transition matrix from the probabilities of all connected devices.

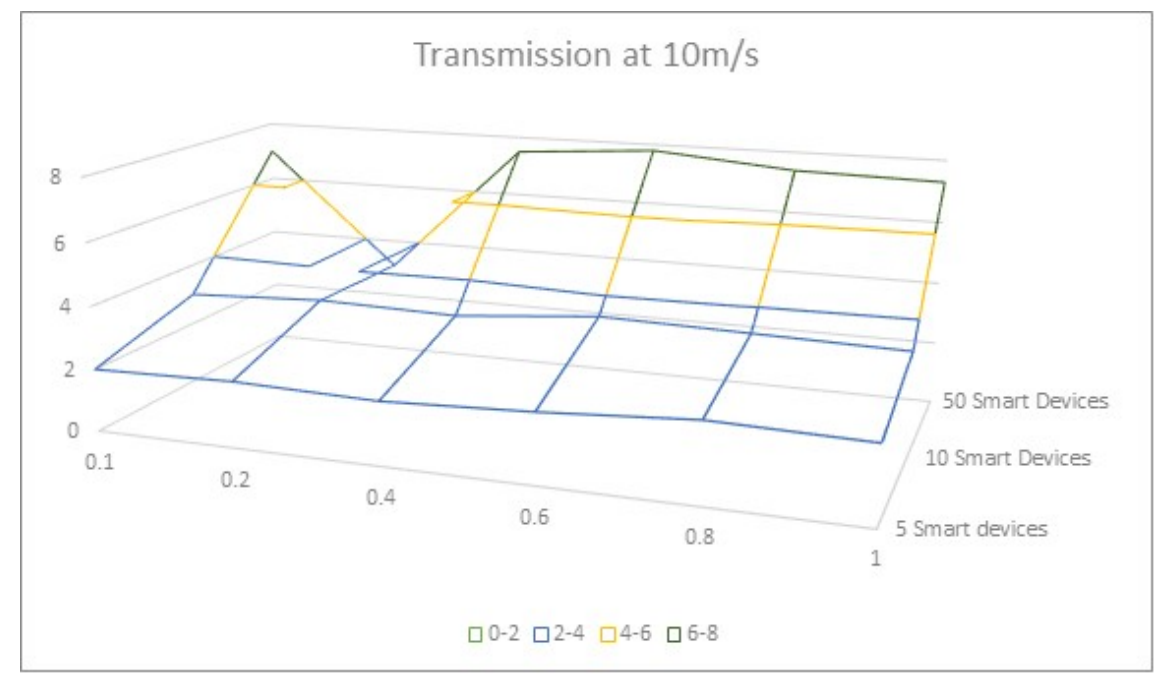

Fig. 6. Transmission in Internet of smart devices at $10 \mathrm{~m} / \mathrm{s}$ 


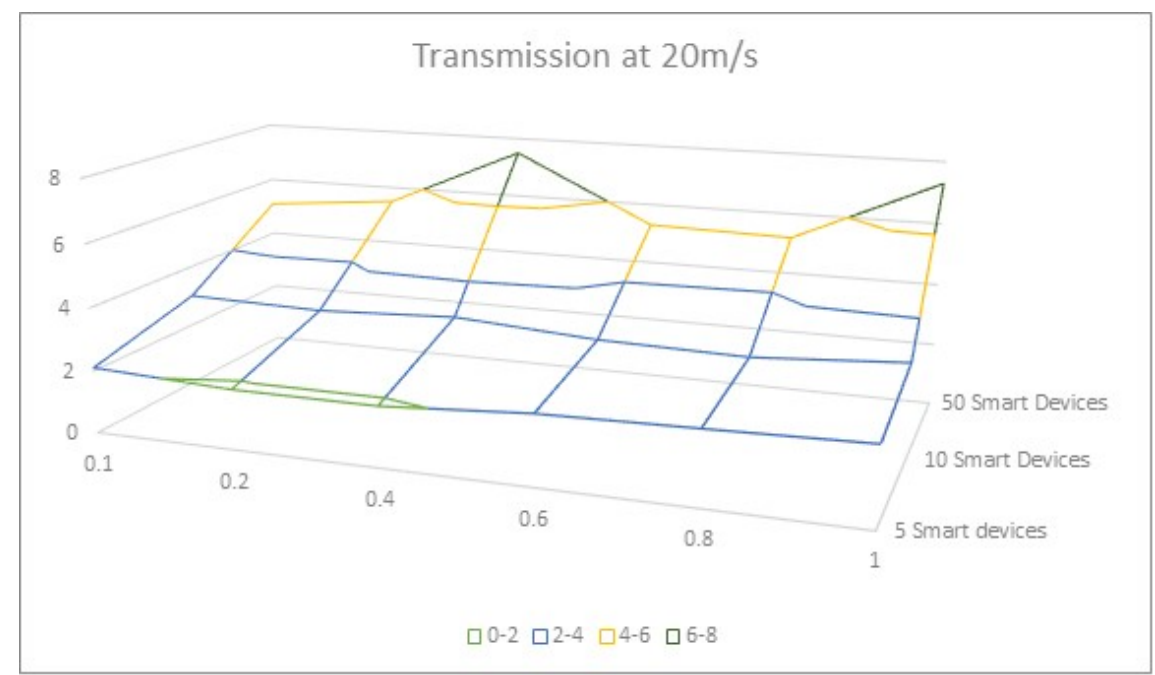

Fig. 7. Transmission in Internet of smart devices at $20 \mathrm{~m} / \mathrm{s}$

Now we will find entropy per symbol row-wise said $H_{1}, H_{2}, H_{3} \ldots \ldots, H_{K}$ according to above transition matrix. After findings of $H_{1}, H_{2}, H_{3} \ldots \ldots, H_{K}$ we will found the whole entropy per symbol of the smart devices.

$\mathrm{H}=\mathrm{H}_{1} \cdot P_{1}+H_{2} \cdot P_{2}+H_{3} \cdot P_{3}+\ldots \ldots \ldots H_{K} \cdot P_{K} \ldots \ldots \ldots \ldots \ldots . .(14)$

We have calculated the velocities of smart devices using Gauss-Markov Mobility Model in the multidimensional area of internet.

In figure 4, R1, R2 and R3 are the reliable devices that are used for establishing a reliable route among internet of smart devices and create a reliable route to evaluate the performance of reliability. The performance evaluation of $\mathrm{R}$ devices among internet of smart devices are calculated (Table 1, fig 8).

Table 1: R-Device Performance comparisons with another device

\begin{tabular}{llllll}
\hline $\begin{array}{l}\text { Devices No of } \\
\text { routes- }\end{array}$ & R-Device & $\begin{array}{l}\text { Normal } \\
\text { Devices }\end{array}$ & Random & No-R & Distributed \\
\hline $\mathbf{2}$ & 0.95 & 0.93 & 0.95 & 0.93 & 0.95 \\
$\mathbf{3}$ & 0.94 & 0.83 & 0.92 & 0.82 & 0.93 \\
$\mathbf{4}$ & 0.92 & 0.74 & 0.85 & 0.75 & 0.90 \\
$\mathbf{5}$ & 0.91 & 0.62 & 0.79 & 0.60 & 0.88 \\
$\mathbf{6}$ & 0.90 & 0.59 & 0.72 & 0.58 & 0.85 \\
$\mathbf{7}$ & 0.88 & 0.55 & 0.70 & 0.53 & 0.82 \\
$\mathbf{8}$ & 0.87 & 0.49 & 0.70 & 0.50 & 0.79 \\
\hline
\end{tabular}

\section{[CHART]}

Fig. 8: R-Device Performance comparisons with another device

The table 2 represents the shortest path between two devices in the internet of smart devices. See fig 9 .

Table 2: R-Device reliability probability comparisons with another device 


\begin{tabular}{llllll}
\hline $\begin{array}{l}\text { Devices No of } \\
\text { routes- }\end{array}$ & R-Device & $\begin{array}{l}\text { Normal } \\
\text { Devices }\end{array}$ & Random & No-R & Distributed \\
\hline $\mathbf{2}$ & 0.88 & 0.7 & 0.83 & 0.72 & 0.82 \\
$\mathbf{3}$ & 0.82 & 0.35 & 0.72 & 0.38 & 0.75 \\
$\mathbf{4}$ & 0.79 & 0.26 & 0.63 & 0.25 & 0.72 \\
$\mathbf{5}$ & 0.75 & 0.15 & 0.54 & 0.17 & 0.70 \\
$\mathbf{6}$ & 0.7 & 0.09 & 0.49 & 0.08 & 0.66 \\
$\mathbf{7}$ & 0.65 & 0.05 & 0.45 & 0.04 & 0.60 \\
$\mathbf{8}$ & 0.64 & 0.07 & 0.42 & 0.05 & 0.54 \\
\hline
\end{tabular}

\section{[CHART]}

Fig. 9: R-Device reliability probability comparisons with another device

The average time taken by the smart device is simulated using our study is shown in the following figure 10 .

Table 3: Average time taken by the smart device

\begin{tabular}{llllll}
\hline $\begin{array}{l}\text { Devices No of } \\
\text { devices- }\end{array}$ & R-Device & $\begin{array}{l}\text { Normal } \\
\text { Devices }\end{array}$ & Random & No-R & Distributed \\
\hline $\mathbf{5}$ & 5 & 28 & 23 & 29 & 7 \\
$\mathbf{1 0}$ & 5 & 40 & 29 & 42 & 9 \\
$\mathbf{1 5}$ & 6 & 45 & 35 & 49 & 12 \\
$\mathbf{2 0}$ & 8 & 49 & 40 & 55 & 15 \\
$\mathbf{2 5}$ & 10 & 54 & 44 & 59 & 16 \\
\hline
\end{tabular}

\section{[CHART]}

Fig. 10: Average time taken by the smart device

\section{Conclusion}

The reliability is the main issue in heterogeneous environment where many sensors, actuators, controllers and intelligent objects etc. are connected to each other. The proposed research had planned to builds research on extending reliability for communication in smart devices using IEEE802.15.4. The main contribution of this research is the creation of the communication framework and provide reliable communication using IEEE802.15.4 among the internet of smart devices. The previous studies have been focused on the creation and optimization the framework for communication, but such research doesn't perform the full framework for reliable communication among internet of smart devices. The proposed framework for internet of smart devices based on IEEE 802.15.4 for the reliable communication to improve the performance of communication reliability is tested and found positive results. The proposed research focused on a framework for providing the communication reliability in the internet of smart devices network. Our main contribution in this study integrates the reliability to the communication framework in internet of smart devices. This study is very useful in Internet of Things framework. The proposed framework has been experimentally implemented. It has predicted well in our comprehensive experiments. The Overall performance of the proposed study in terms of device to device delay and the communication reliability has been evaluated.

\section{References}

1. Gummadi, P. Krishna, et al. "Improving the Reliability of Internet Paths with One-hop Source Routing." OSDI. Vol. 4. 2004.

2. Wu, Geng, et al. "M2M: From mobile to embedded internet." IEEE Communications Magazine 49.4 (2011). 
3. $\mathrm{Li}, \mathrm{Xu}$, et al. "Smart community: an internet of things application." IEEE Communications Magazine 49.11 (2011).

4. Sharma, Abhilash, Tanweer Alam, and Dimpi Srivastava. "Ad Hoc Network Architecture Based on Mobile Ipv6 Development." Advances in Computer Vision and Information Technology (2008): 224.

5. Alam, Tanweer, and Mohammed Aljohani. "Design and implementation of an Ad Hoc Network among Android smart devices." Green Computing and Internet of Things (ICGCIoT), 2015 International Conference on. IEEE, 2015.

6. Moslehi, Khosrow, and Ranjit Kumar. "A reliability perspective of the smart grid." IEEE Transactions on Smart Grid 1.1 (2010): 57-64.

7. Alam, Tanweer. "Middleware Implementation in Cloud-MANET Mobility Model for Internet of Smart Devices." IJCSNS 17.5 (2017): 86.

8. Metke, Anthony R., and Randy L. Ekl. "Smart grid security technology." Innovative Smart Grid Technologies (ISGT), 2010. IEEE, 2010.

9. Katasonov, Artem, et al. "Smart Semantic Middleware for the Internet of Things." Icinco-Icso 8 (2008): 169-178.

10. Gao, Jingcheng, et al. "A survey of communication/networking in smart grids." Future Generation Computer Systems 28.2 (2012): 391-404.

11. Militano, Leonardo, et al. "Device-to-device communications for $5 \mathrm{G}$ internet of things." EAI Endorsed Transactions on Internet of Things 15.1 (2015): 1-15.

12. Alam, Tanweer, and Mohammed Aljohani. "Design a new middleware for communication in ad hoc network of android smart devices." Proceedings of the Second International Conference on Information and Communication Technology for Competitive Strategies. ACM, 2016.

13. Aljohani, Mohammed, and Tanweer Alam. "Design an M-learning framework for smart learning in ad hoc network of Android devices." Computational Intelligence and Computing Research (ICCIC), 2015 IEEE International Conference on. IEEE, 2015.

14. Stergiou, Christos, et al. "Secure integration of IoT and cloud computing." Future Generation Computer Systems 78 (2018): 964-975.

15. Al-Kuwaiti, Mohamed, Nicholas Kyriakopoulos, and Sayed Hussein. "A comparative analysis of network dependability, fault-tolerance, reliability, security, and survivability." IEEE Communications Surveys \& Tutorials 11.2 (2009).

16. Sharma, Vibhu Saujanya, and Kishor S. Trivedi. "Architecture based analysis of performance, reliability and security of software systems." Proceedings of the 5th International Workshop on Software and Performance. ACM, 2005.

17. Aljohani, Mohammed, and Tanweer Alam. "Real time face detection in ad hoc network of android smart devices." Advances in Computational Intelligence. Springer, Singapore, 2017. 245-255.

18. Alam, Tanweer. "Fuzzy control based mobility framework for evaluating mobility models in MANET of smart devices." ARPN Journal of Engineering and Applied Sciences 12.15 (2017): 4526-4538.

19. Bermolen, Paola, et al. "Estimating the transmission probability in wireless networks with configuration models." ACM Transactions on Modeling and Performance Evaluation of Computing Systems 1.2 (2016): 9.

20. Kumar, Vijay, et al. "Markov model for reliable packet delivery in Wireless Sensor Networks." International Journal of Computer Science Issues 8.3 (2011): 1694-0814.

21. Aljohani, Mohammed, and Tanweer Alam. "An algorithm for accessing traffic database using wireless technologies." Computational Intelligence and Computing Research (ICCIC), 2015 IEEE International Conference on. IEEE, 2015.

22. Alam, Tanweer, and Mohammed Aljohani. "An approach to secure communication in mobile adhoc networks of Android devices." Intelligent Informatics and Biomedical Sciences (ICIIBMS), 2015 International Conference on. IEEE, 2015.

23. Heinzelman, Wendi Rabiner, Anantha Chandrakasan, and Hari Balakrishnan. "Energy-efficient communication protocol for wireless microsensor networks." System sciences, 2000. Proceedings of the 33rd annual Hawaii international conference on. IEEE, 2000. 
24. Koetter, Ralf, and Muriel Médard. "An algebraic approach to network coding." IEEE/ACM Transactions on Networking (TON) 11.5 (2003): 782-795.

25. Li, Fangxing, et al. "Smart transmission grid: Vision and framework." IEEE transactions on Smart Grid 1.2 (2010): 168-177.

26. Gungor, Vehbi C., et al. "Smart grid technologies: Communication technologies and standards." IEEE transactions on Industrial informatics 7.4 (2011): 529-539.

27. Singh, Parbhakar, Parveen Kumar, and Tanweer Alam. "Generating Different Mobility Scenarios in Ad Hoc Networks.", International Journal of Electronics Communication and Computer Technology, 4(2), 2014

28. Alam, Tanweer, and B. K. Sharma. "A New Optimistic Mobility Model for Mobile Ad Hoc Networks." International Journal of Computer Applications 8.3 (2010): 1-4.

29. Alam, Tanweer, and Tapesh Kumar Tyagi. "A Random Waypoint Model for Mobility in Ad Hoc Network Simulation Area." Journal of Advanced Research in Computer Engineering 3.1 (2009): 13-17.

30. Alam, Tanweer, et al. "Scanning the Node Using Modified Column Mobility Model." Computer Vision and Information Technology: Advances and Applications (2010): 455.

31. Bello, Oladayo, and Sherali Zeadally. "Intelligent device-to-device communication in the internet of things." IEEE Systems Journal 10.3 (2016): 1172-1182. 